\title{
A Multi-Scale Consideration of Daylight in a Real Urban Context
}

\author{
Antoine Bugeat ${ }^{1}$, Eduardo Fernández ${ }^{2}$, Benoit Beckers ${ }^{1}$, José Aguerre ${ }^{2}$ \\ ${ }^{1}$ Urban Physics Joint Laboratory, Université de Pau et des Pays de l'Adour, E2S UPPA, Campus \\ Montaury, 64600, Anglet, France \\ ${ }^{2}$ Centro de Cálculo, Universidad de la República. J. H. y Reissig 565, 11300, Montevideo, Uruguay
}

\begin{abstract}
In dense urban environments, many indoor spaces are poorly daylighted. This article presents the impact of the optical characteristics of facade elements on a set of buildings located in a historic city centre street. Climate Based Daylight Modelling (CBDM) metrics at a street scale provide accurate information on the daylight performance of buildings interiors. The results show that the daylight autonomy (DA) of dark spaces is strongly influenced by the reflectance of the facades of surrounding buildings. The radiosity method presented allows for an accurate and fast calculation of CBDMs on a large number of sensors for a multitude of facade configurations with different reflectances. More detailed knowledge on the importance and potential of light interreflection in a dense urban environment is presented.
\end{abstract}

\section{Introduction}

One of the major challenges in architecture and urban planning is to design spaces in order to distribute natural light as effectively as possible. In dense urban areas, ensuring a minimum level of daylight availability for all living and working areas is a difficult task for architects and engineers. Many research studies propose methods to help designers in this task (Mardaljevic, 2012; Compagnon, 2004). Shape and composition of the facades and external elements are the two main properties that have a significant impact on the distribution of solar radiation (Strømann-Andersen and Sattrup, 2011). However, in an already built historic city center, the possibilities of changing geometric parameters such as the shape or orientation of streets are reduced or non-existent. The simplest and cheapest way to do this is to change the composition of the street surfaces. To quantify the daylight performance of building's interior, CBDM metrics have become reference methods in recent years, and are used in building guidelines and regulations (IESNA, 2012). They allow an accurate assessment of daylight over an entire year, taking into account the dynamic nature of light conditions in relation to time, season and climate. The use of CBDM metrics is usually limited to a building scale, where the interest of the study is focused on the performance of a single project. Recent research suggests effective methods to calculate these indicators at urban scale (Dogan, 2012). However, these methods can involve long simulations, and important assumptions about the model and the distribution of light in indoor spaces must be made.

The objective of this study is to quantify, on a set of buildings in a street of a dense urban environment, the importance of the changes produced by the modification of the optical characteristics of the external elements on the daylight performance of interior spaces. This is a multi-scale problem where decisions made at street level are analyzed at a building scale. The case studied is a street in the historic city center of Bayonne in the French Basque Country. To be able to test a multitude of configurations with different reflectances in a reasonable time, simulations are performed using a method based on radiosity algorithms (Fernandez, 2016).

\section{Simulation Method}

The daylight received at a point is always composed of a direct part, coming straight from the sky dome and the Sun, and a reflected part, coming from the reflections occurring between the surfaces of the environment. The ratio between direct and reflected parts varies according to the configuration of the area. The more unobstructed is the environment, the larger the direct component. On the other hand, the denser the environment, the more important is the reflected component in the total illumination. Urban environments, as the one studied here, are dense environments. It is therefore important to consider the reflections with precision in this study. The radiosity method (Goral, 1984) allows to accurately calculate reflections between diffuse surfaces and is the one used in this study.

\section{The model}

The model was built following a 3D measurement campaign in order to perform multi-physical simulations of finite elements on an urban scale (Acuña Paz y Miño, 2018). Radiosity requires a meshed model with surface elements called patches. The model for this study consists of a total of 67,056 patches. 59,392 patches with an average surface area of $0.27 \mathrm{~m}^{2}$ constitute the city environment, i.e. the inside and outside of the buildings, the road surface and the surrounding buildings.

The 3D survey shows singular characteristics of the street such as arcade walkways on each side for pedestrian access. The two façades face North (North Façade) and South (South façade). This street has an average height-to-width ratio $\mathrm{H} / \mathrm{W}$ equal to 2 which corresponds to a narrow street, typical of older districts. 

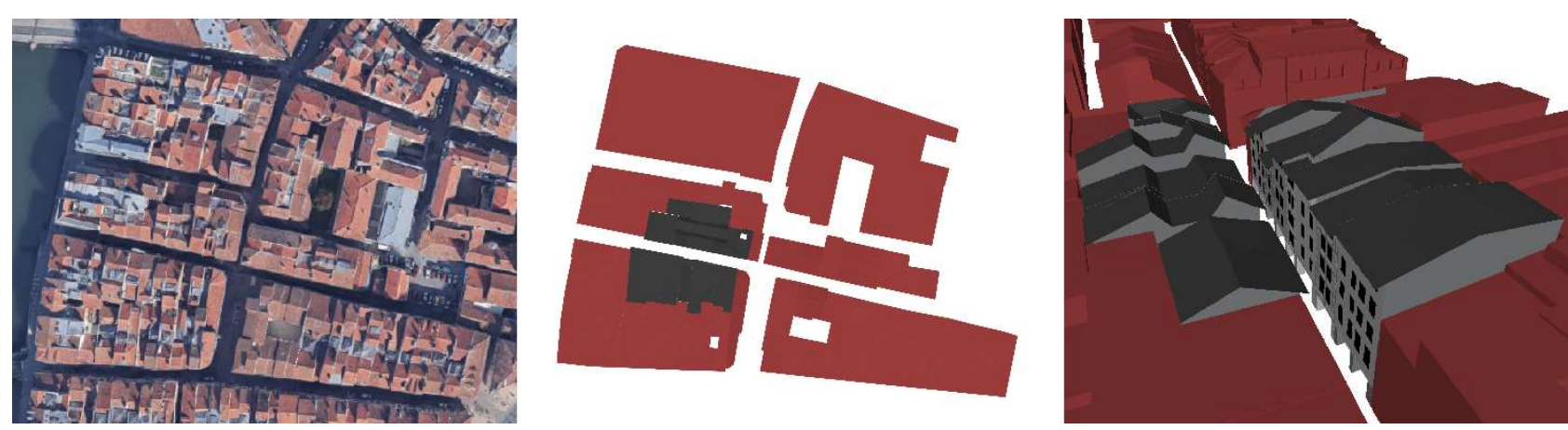

Figure 1: Satellite image (left), top view of the model (middle) and perspective view of the model (right). The set of buildings studied are gray and the surrounding buildings are red.

Each building has a different height that varies between 13 and $17 \mathrm{~m}$. The size and number of floors also varies between 3 and 4 according to each building, in the same way as the number and size of openings. Only the interior layout has been simplified due to a lack of information. Hypotheses for the interiors were taken based on plans of a typical apartment in the city. All the rooms studied face directly onto the street and have an identical depth of $6 \mathrm{~m}$. The surfaces that make up the interiors have a reflection coefficient of: $30 \%$ for the floor, $70 \%$ for the walls and $80 \%$ for the ceiling. The light transmission of the openings is $80 \%$. The street ground has a reflectance of $30 \%$. These characteristics remain constant during this study.

\section{Daylight Coefficient and CBDM metrics}

Climate Based Daylight Modeling metrics such as Daylight Autonomy (DA), Spatial Daylight Autonomy (sDA), Useful Daylight Illuminance or Total Annual Illuminance (TAI) are obtained from a dynamic simulation. DA and TAI are calculated in this paper. DA is the percentage of time during a year when daylight provides illumination above a threshold. Two thresholds are used, according to the literature for work and residential spaces: 150 and 300 lux (CIBSE/SLL, 2013; Paule, 2015). This type of indicator requires predicting the hourly illuminances received on the sensors of a virtual surface called a work plane located above the ground. A few thousand sky conditions, modeled from measured and standardized climate data, must be considered. The principle of Daylight Coefficients (DC) consists in dividing the sky into patches and calculating the contribution of each patch to the illumination of each sensor (Tregenza and Waters, 1983). The illuminance of a sensor for a given sky condition can then be obtained by adding the product between the luminance and DC for each sky patch (1).

$$
I_{s}=\sum_{j=1}^{k} D_{s, j} L_{j} \Delta \omega_{j}
$$

Where $I_{s}$ is the illuminance value of sensor $s$ [lux], $D_{s, j}$ is the daylight coefficient for sensor $s$ and sky patch $j, L_{j}$ is the luminance of sky patch $j\left[\mathrm{~lm} / \mathrm{m}^{2} / \mathrm{sr}\right], \Delta \omega_{j}$ is the solid angle of sky patch $j$ [sr]. This method then makes it possible to take into account several thousand sky conditions while performing only one simulation. It is one of the most used methods in daylight simulation software.

In this study, the sky dome is discretized according to the Reinhart model, which is a subdivision of the Tregenza (1987) model composed of 2305 elements (144 $\mathrm{x} 4+1)$. The weather data comes from the epw file of the nearby city of Bordeaux (Crawley, 1999) and sky luminance models is built according to All-Weather Model of Perez (1993). Additionally, there are 5348 sensors representing the work planes located in the interiors of each building.

\section{Daylight Coefficient with Radiosity}

DC and radiosity have one common point: the patch discretization of the sky dome. The principle of daylight coefficients is therefore suited to radiosity algorithms (Geebelen, 2005). The traditional radiosity consists in dividing each surface of the scene into several polygons called "patches". The exchanges between each patch are efficiently determined by a discrete formulation of the global illumination equation assuming that the patches emit and/or reflect in a perfectly diffuse way. The view factors are structured as a square matrix of dimension 67,056 . For each patch, they provide information on the proportion of energy received from each of the other patches. These view factors depend only on geometry and are obtained with the hemicube technique (Cohen and Greenberg, 1985). The radiosity in each patch is calculated by solving a system of equations (Goral, 1984). The same principle applies to the corresponding photometric quantities (Equation 2). In this way, it is also possible to obtain the illuminance values in each patch (Equation 3) (Beckers, 2013).

$$
\begin{aligned}
B & =\mathbf{M}_{\text {rad }} E, \text { with } \mathbf{M}_{\text {rad }}=(\mathbf{I}-\mathbf{R F})^{-1} \\
I & =\mathbf{M}_{\text {ill }} \mathbf{F} E, \text { with } \mathbf{M}_{\text {ill }}=(\mathbf{I}-\mathbf{F R})^{-1}
\end{aligned}
$$

Where $B$ is the vector of luminous flux values emitted or reflected per unit area in $\left[1 \mathrm{~m} / \mathrm{m}^{2}\right]$ (equivalent to radiosity in radiometry), $I$ is the vector of illuminance values in [lux] (equivalent to irradiance in radiometry), $\mathbf{I}$ is the identity matrix, $\mathbf{R}$ is the diagonal matrix of diffuse reflectance values, $\mathbf{F}$ is the square matrix of view factors, $\mathbf{M}_{\text {rad }}$ is the matrix of interaction coefficient for the radiosity equation, $\mathbf{M}_{\boldsymbol{i l l}}$ is the matrix of interaction coefficient for the illuminance equation and $E$ is the vector of luminous exitance values in $\left[\mathrm{lm} / \mathrm{m}^{2}\right]$ 
(equivalent to exitance in radiometry). Only the illuminance received on the sensors is required for the calculation of CBDM metrics.

The illuminance of the sensors can be divided into two parts: $I_{s, d i r}$. and $I_{s, r e f l}$. Furthermore, the matrix $\mathbf{F}$ is divided in the following way:

$$
\mathbf{F}=\left[\begin{array}{lll}
\mathbf{F}_{c c} & \mathbf{F}_{c s} & \mathbf{F}_{c \mathrm{k}} \\
\mathbf{F}_{s c} & \mathbf{F}_{s s} & \mathbf{F}_{s k} \\
\mathbf{F}_{k c} & \mathbf{F}_{k s} & \mathbf{F}_{k k}
\end{array}\right]
$$

City patches are identified with $c$ sub-indices, sensors patches with $s$, and sky patches with $k$. For example, $\mathbf{F}_{s c}$ is the view factor matrix of sensor patches seeing city patches and $\mathbf{F}_{c c}$ is the view factor matrix of city patches seeing city patches. Only $\mathbf{F}_{c c}, \mathbf{F}_{c \mathrm{k}}, \mathbf{F}_{s k}$ and $\mathbf{F}_{s c}$ are required.

$I_{s, \text { dir. }}$ is the vector of illuminance values of the sensors coming directly from the sky [lux]:

$$
I_{s, \text { dir. }}=\mathbf{F}_{s k} E_{k}
$$

where $\mathbf{F}_{s k}$ is the view factor matrix of sensor patches seeing sky patches, and $E_{k}$ is the vector of luminous exitance values of the sky patches in $\left[1 \mathrm{~m} / \mathrm{m}^{2}\right]$.

$I_{s, \text { refl. }}$ is the vector of illuminance values of the sensors, which is the radiosity of the environment that reaches the sensors.

$$
\begin{gathered}
I_{s, r e f l .}=\mathbf{F}_{s c} B_{c} \\
\text { with } B_{c}=\mathbf{K}_{c k} E_{\boldsymbol{k}} \\
\text { and } \mathbf{K}_{c k}=\left(\mathbf{I}-\mathbf{R}_{c} \mathbf{F}_{c c}\right)^{-1} \mathbf{R}_{c} \mathbf{F}_{c k}
\end{gathered}
$$

where, $\mathbf{F}_{s c}$ is the view factor matrix of sensor patches seeing city patches, $\mathbf{F}_{c c}$ is the view factor matrix of city patches seeing city patches, $\mathbf{F}_{c k}$ is the view factor matrix of city patches seeing sky patches, $\mathbf{K}_{c k}$ is the matrix of interaction coefficient only between city patches and sky patches, $\mathbf{K}_{c k}(\mathrm{i}, \mathrm{j})$ is the proportion of flux emitted by sky patch $\mathrm{j}$, that is reflected by city patch $\mathrm{i}$ and $\mathbf{R}_{c}$ is the diagonal matrix of diffuse reflectance values of city patches. The illuminance of the sensors for a given sky condition is then obtained after a simple multiplication of matrices. Then, the illuminance depends only on the luminous exitance of the sky patches and it is similar to the daylight coefficient principle:

$$
I_{s}=\left(\mathbf{F}_{s c} \mathbf{K}_{c k}+\mathbf{F}_{s k}\right) E_{k}
$$

To obtain $\mathbf{K}_{c k}$, instead of calculating the inverse of $\left(\mathbf{I}-\mathbf{R}_{c} \mathbf{F}_{c c}\right)$, the linear system $\left(\left(\mathbf{I}-\mathbf{R}_{c} \mathbf{F}_{c c}\right) \mathbf{R}_{c}\right) \mathbf{K}_{c k}=$ $\mathbf{F}_{c k}$ is solved iteratively (Aguerre, 2017). In this approach, $\left(\mathbf{I}-\mathbf{R}_{c} \mathbf{F}_{c c}\right) \mathbf{R}_{c}$ is a sparse matrix, which allows to solve the system in any personal computer. With this method, the number of light reflections is limited to a certain number. Since ray tracing algorithms also limit the number of reflections, this allows the same calculation assumptions to be used to make an accurate comparison with the results obtained with RADIANCE (Ward 1994).

\section{Specular Reflection with Extended View factor}

The traditional radiosity method involves an environment composed exclusively of surfaces that reflect light in a diffuse way. Fortunately, in urban areas there are mainly diffuse surfaces. However, buildings with outer envelopes made of materials that produce specular reflections are nowadays more common. This can lead to a change in the distribution of natural light on the environment. With the presence of surfaces that reflect light in a specular way, the radiosity equation is no longer applicable. Several works have been carried out to adapt the radiosity method to include other types of reflection. In most research, the BRDF (Bidirectional Reflectance Distribution Function) model of each surface is decomposed into a linear combination of perfectly diffuse and perfectly specular reflection (Rushmeier and Torrance, 1990). Among all the existing methods for taking into account specular reflections in radiosity, one of the most effective is the extended view factor method (Sillion and Puech, 1989). Typically, the view factor $\mathbf{F}(i, j)$ is the fraction of total energy leaving patch $\mathrm{i}$ and arriving patch $\mathrm{j}$ directly, i.e. without diffuse or specular reflection. The principle of extended view factors is to consider surfaces with specular reflection as additional paths by which light can reach other patches. Thus, two patches that see each other by specular reflection through one or more "mirror" patches have an additional specular view factor (S) representing the fraction of energy that is transferred through these paths:

$$
\mathbf{F}_{\text {eff }}=\mathbf{F}+\mathbf{S}
$$

where $\mathbf{F}_{\text {eff }}$ is the extended view factor matrix. $\mathbf{S}$ can be calculated by different methods. Sillion and Puech (1989) propose to use a ray tracing method to calculate $\mathbf{S}$. Once the extended view factor matrix $\mathbf{F}_{\text {eff }}$ is obtained, the radiosity solution can be calculated in the same way by solving the system of equations describing the exchanges between diffuse surfaces, with the difference that conventional view factors are replaced by extended view factors. Exchanges by specular reflections are therefore taken into account as they are included in the extended view factors.

\section{Results}

\section{Influence of exterior reflectance}

In Figure 2, $\mathrm{DA}_{150}$ maps are represented for all the studied buildings in 3 configurations with different reflectances of the exterior walls. The case A in Figure 2 corresponds to the existing case with the reflectances measured in situ, with an average reflectance of $52 \%$ of the exterior walls. The case $\mathrm{B}$ corresponds to clear facades with a reflectance of $80 \%$. This configuration is equivalent to a street composed of walls painted in white. The case $\mathrm{C}$ consists of dark facades with a reflectance of $20 \%$, representing walls made of red brick or dark-colored walls. These two other scenarios were chosen to study two opposing cases representing realistic configurations. To allow a spatial interpretation of the results, all the maps of each floor are presented together. The work surfaces are centered under the diagram of their respective building facades and their vertical position is proportional to their actual height in the model. The differences between the overall 


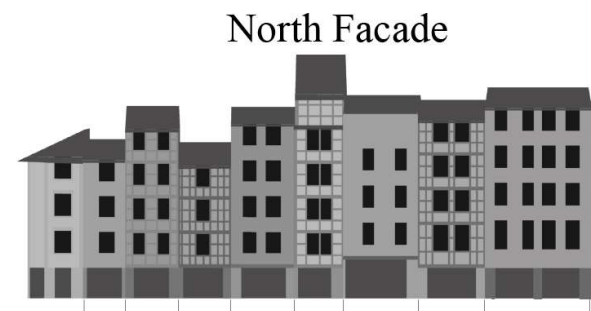

A.Reflectance measured on-site Facade walls reflectance $=52 \%$

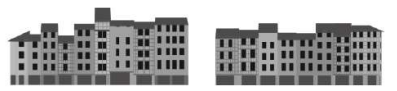

\section{B. High reflectance facades}

Facade walls reflectance $=80 \%$

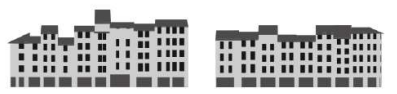

\section{C.Low reflectance facades}

Facade walls reflectance $=20 \%$

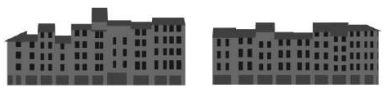

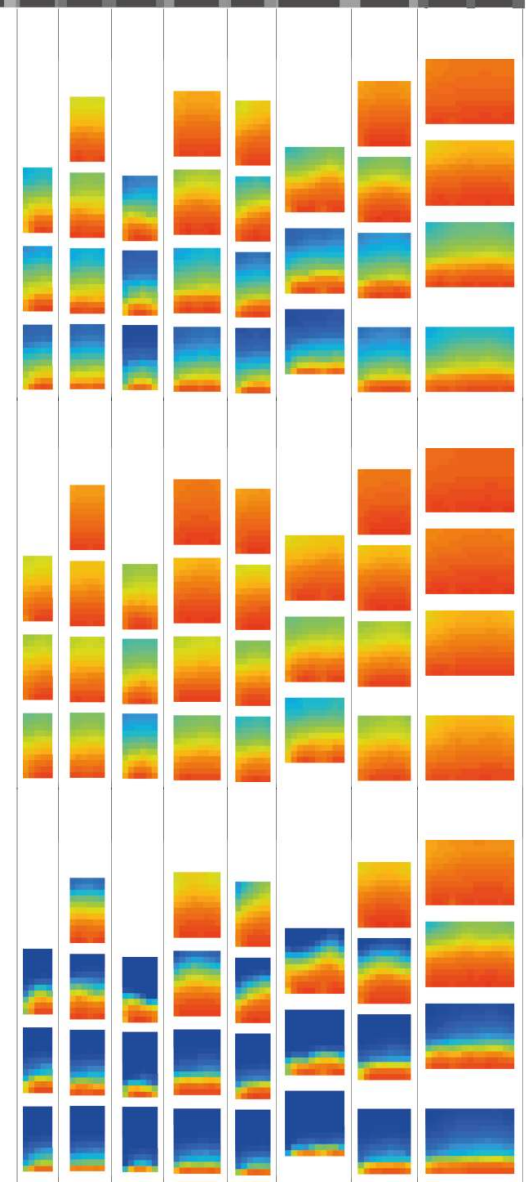

\section{South Facade}
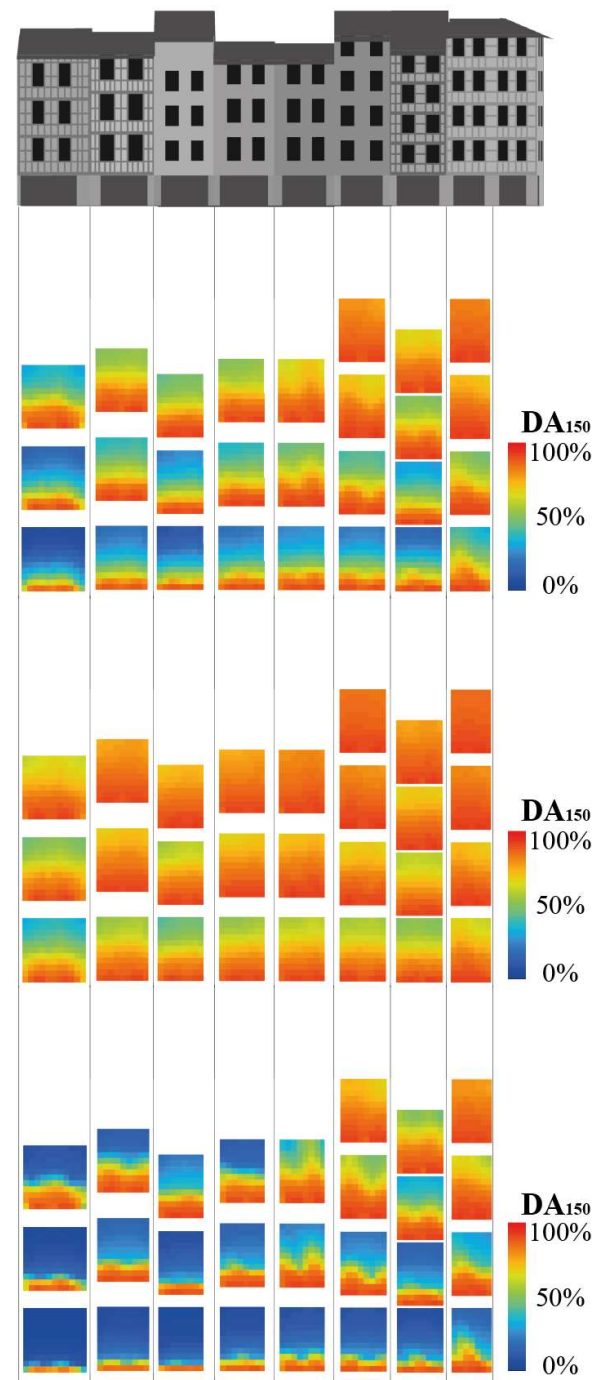

Figure 2: DA 150 maps of all buildings of the street studied with different facade reflectance configurations. Case A: Mean reflectance measured on-site (52\%). Case B: High reflectance (80\%). Case C: Low reflectance (20\%).

performances of the 3 cases exposed are clearly visible. The differences between the North and South facades are almost non-existent in all 3 cases. This phenomenon is explained at the end of the section with Figure 4. Due to the geometric characteristics of the street buildings, natural light is not distributed in the same way in all the rooms. Some parts, with the same orientation and at the same height, can achieve substantially different levels of performance. This highlights the sensitivity of indicators to a multitude of geometric factors. These geometric factors can be size and position of openings, the dimensions of interiors or masks created by neighboring buildings. The asymmetric distribution of light on the work planes is also due to these same characteristic of the model. For these reasons, it is difficult to compare rooms or buildings individually.

For a simpler and more comprehensive reading, the results are grouped according to each floor in Figures 3 and 4. In Figure 3, the evolution of the median $\mathrm{DA}_{150}$ and median $\mathrm{DA}_{300}$ of each floor are represented as a function of the reflectance of the exterior walls. The North and South facades are merged. There are large inequalities between the upper and lower floors for cases with low reflectances. The greater the reflectances of the exterior walls, the more these inequalities tend to be reduced. For a facade reflectance of $20 \%$, the difference in $\mathrm{DA}_{150}$ between the first and last floor is $67 \%$. The difference in $\mathrm{DA}_{150}$ between the first and last floor in the case of an $80 \%$ reflectance of the facades is only $22 \%$. The impact of the reflectance of the facades is especially important for the lower floors with a limited direct view of the sky. In order to quantify the contributions due to the reflections of the facades, it is possible to compare the results in $\mathrm{DA}_{150}$ with those obtained for a reflectance of $0 \%$, equivalent to perfectly black walls. This is an unrealistic reflectance value but it allows studying the case where reflections from facades are not considered. For cases $\mathrm{A}$ and $\mathrm{B}$ on the first floor, the contribution of the reflections of the facades on the $\mathrm{DA}_{150}$ results is respectively $34 \%$ and $59 \%$. These gains due to the reflections of the facades represent $87 \%$ and $92 \%$ of the 


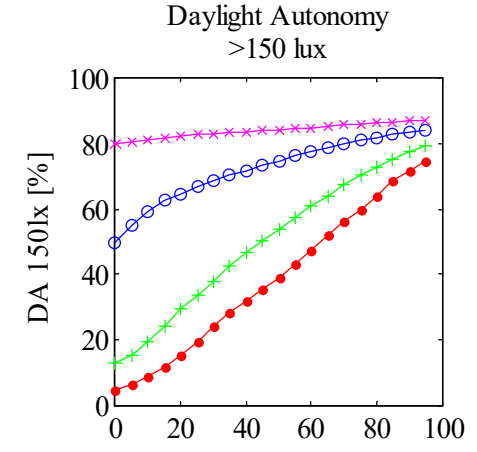

Reflectance of Exterior Walls [\%]

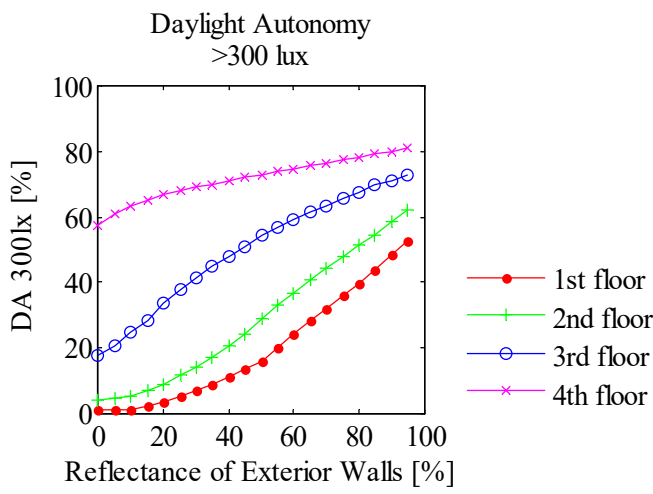

Figure 3: Impact of facade reflectance on the median $D A_{150}$ (up) and the median $D A_{300}$ (down) of each floor.
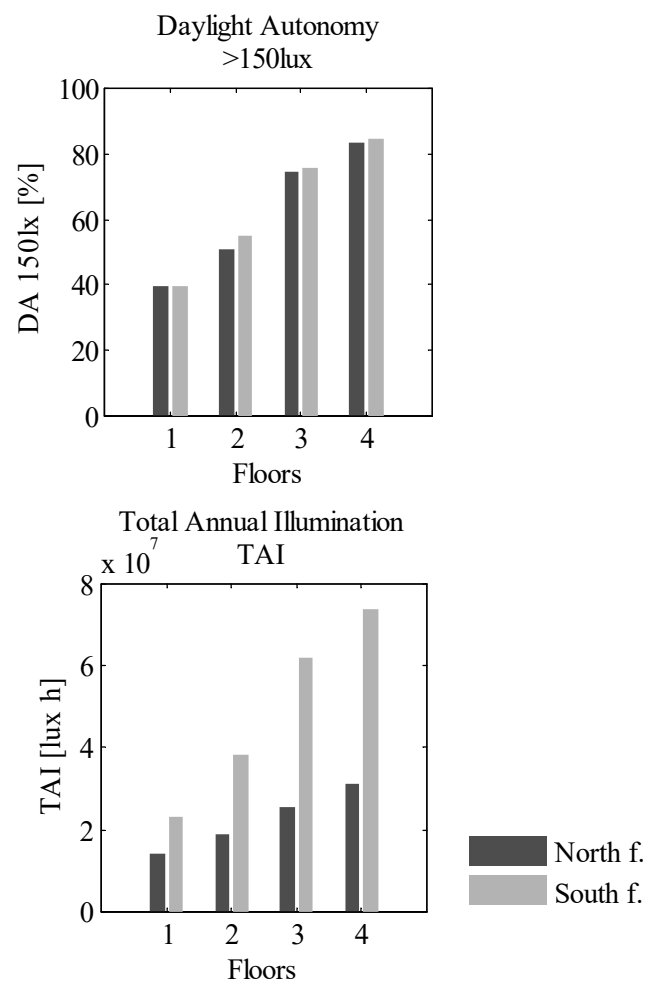

Figure 4: $D A_{150}$ (up) and Total Annual Illuminance incident on windows (down) for each floor of North and South facing buildings with measured reflectances (mean 52\%) of the street facades. total $\mathrm{DA}_{150}$. This highlights the importance of the part reflected in urban areas on potential energy savings. DA are very sensitive to reflectances, which emphasizes the importance of providing accurate input reflectances for simulations (Brembilla, 2016).

In Figure 4, the $\mathrm{DA}_{150}$ values calculated inside buildings and the TAI values incident on the windows are presented for the measured reflectances case. These two climate based indicators were chosen because their calculation principles are different. The first is calculated internally on a work plane and was not originally used for urban studies. It has a calculation criterion to estimate the time required by the user to use artificial lighting according to a horizontal illumination threshold value, here chosen at 150 lux. The second is calculated outside the buildings, which shows the cumulative illumination received throughout the year. It is usually this type of indicator that is used to analyze the performance or impact of a project on an urban scale. The needs in terms of modeling and calculation are less important than for the calculation of DA type indicators because fewer sensors are required and the interior is not taken into account. Only the outer envelope of the buildings is required. As seen in Figure 2, the $\mathrm{DA}_{150}$ differences between buildings on the south and north facades are almost non-existent. For TAI, the pattern is different: the southern facade receives significantly more radiation, about twice as much as the northern facade. This difference in trend between the two indicators is explained by the notion of threshold present in the calculation of DA. In clear sky conditions, lighting levels is significantly higher on the south side than on the north side due to direct sunlight, so TAI in the south is logically higher than in the north. Thanks to interreflections between the facades, the light received in the buildings on the north facade can nevertheless exceeds the DA threshold of 150 lux and so satisfy the lighting needs of users. South-facing buildings reach light levels significantly higher than those of North-facing buildings but both of them can perceive satisfactory daylight conditions. This notion of threshold based on indoor illuminance criteria is a key element to give relevant information on the daylight performance of buildings.

\section{Influence of one building façade}

Figure 5 shows the $\mathrm{DA}_{150}$ losses due to the change in the façade characteristics of a single building over all the neighboring buildings. The purpose of the two cases investigated is to simulate an eventual renovation of the facade of a single building based on the existing case (average facade reflectance of $52 \%$ ). In case $\mathrm{D}$, the modified façade is darkened to a diffuse reflectance of $20 \%$. In case E, the modified façade is also darkened to a reflectance of $20 \%$, with the difference that it reflects in a perfectly specular way. This corresponds to a glass facade or a dark metal cladding. The losses are focused on the two buildings located opposite to the modified facade while the $\mathrm{DA}_{150}$ performance of the rest of the street is unchanged. This characterizes the local aspect of the impact of facade reflectances. Nevertheless, the most 

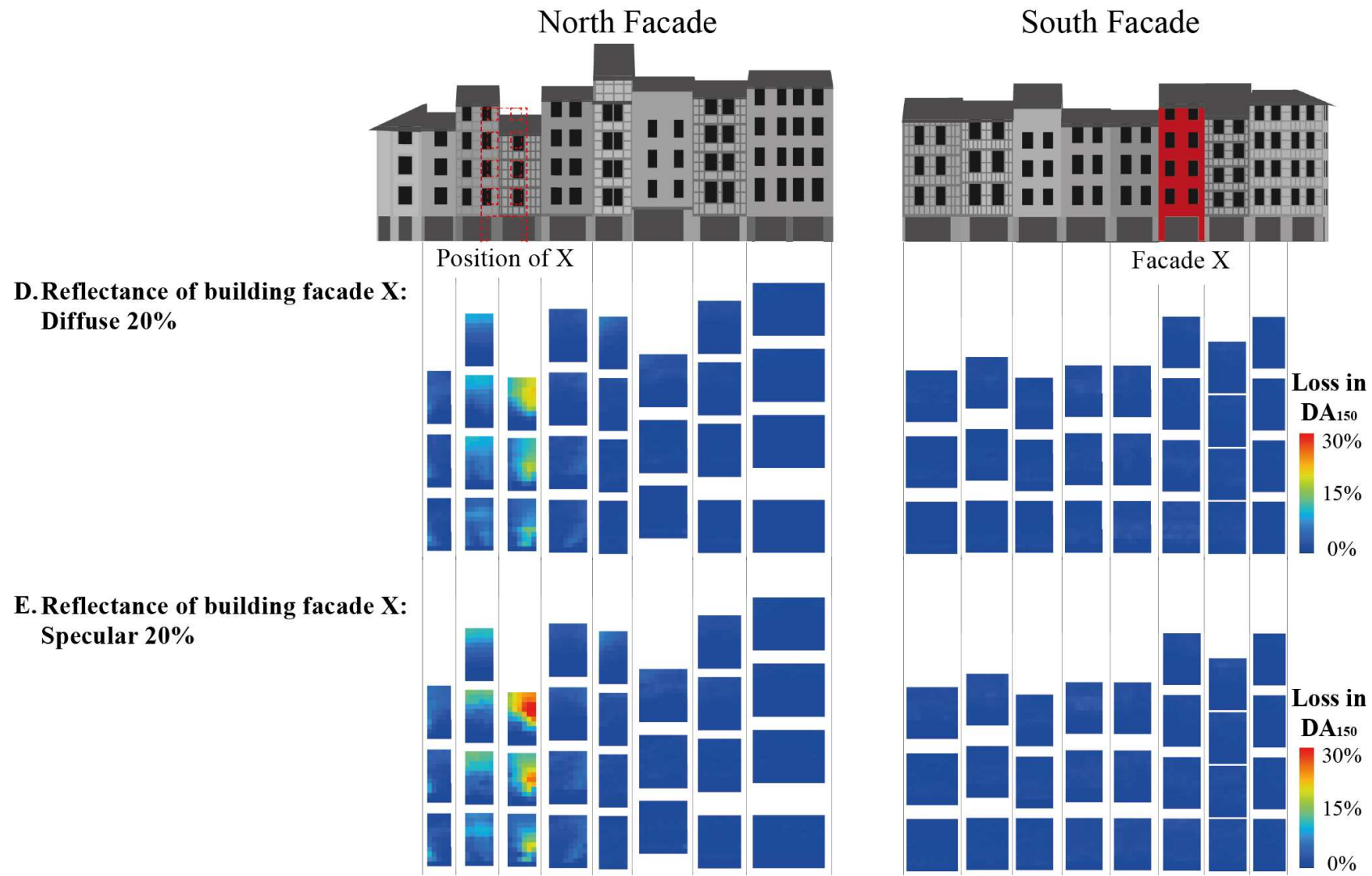

Figure 5: Loss in $D A_{150}$ due to the change in the characteristics of facade X (red) from the actual case $(52 \%$ reflectance). Case D: diffuse reflectance of façade X: 20\%. Case E: specular reflectance of façade X: $20 \%$.

affected building loses on average $7 \%$ for case $\mathrm{D}$ and $11 \%$ for case $\mathrm{E}$ on its initial level of $\mathrm{DA}_{150}$, even if the modified façade reflects the same amount of light $(20 \%)$ In cases $\mathrm{D}$ and $\mathrm{E}$, the $\mathrm{DA}_{150}$ are still lower for the specular case. When the facade is specular, the light is reflected in a single direction. The reflected light is then concentrated in a small area and the surrounding areas are neglected. On a sunny day, the Sun's rays can reach the inside of a building after specular reflection, producing strong localized illuminances. However, this requires the Sun to be in a certain direction that may only happen a few times during a day and a year. When this is not the case, the rays of the Sun arriving on the specular facade can be redirected away from the scene, thereby obscuring it.

\section{Validation and analysis of the method}

To validate the results obtained with the proposed radiosity method, a comparison with the results obtained using RADIANCE was performed. The calculation assumptions, such as the geometry of the model, the characteristics of the materials or the definition of the sky are identical in both methods. The radiosity results demonstrated the importance of the reflected part in interior spaces receiving low daylight levels, therefore special attention was given to the number of reflections taken into account in the simulations. Like ray-tracing algorithms, the radiosity method offers the possibility of limiting the number of reflections. This very important parameter is therefore carefully set to the same number for both methods. The 2-Phase method was used with the RADIANCE rcontrib command with the following simulation parameters: -ab 10 -ad 500000 -lw 1e-8. Table 1 shows the average absolute error (MAE) (Equation 9, where $n$ is the total number of sensors), the standard deviation (STD) of the absolute errors and the quantile $90 \%$ of the absolute errors for the $\mathrm{DA}_{150}$ obtained for the 5 cases A, B, C, D and E (same cases shown in Figures 2 and 5). RADIANCE results are considered as the exact values to compute the absolute errors. However, the Radiosity and RADIANCE results are both approximations. Two identical simulations done with Radiance gave an MAE $=0.2 \%$ due to the Monte Carlo methods.

Table 1: Mean Absolute Error between DA 150 , STD of the absolute errors and Quantile $90 \%$ of the absolute errors obtained between RADIANCE and the proposed radiosity method.

\begin{tabular}{|c|c|c|c|}
\hline CASE & MAE & STD & Quant. 90\% \\
\hline A & $0.55 \%$ & $0.66 \%$ & $1.25 \%$ \\
\hline B & $0.54 \%$ & $0.53 \%$ & $1.20 \%$ \\
\hline C & $1.01 \%$ & $1.15 \%$ & $2.47 \%$ \\
\hline D & $0.57 \%$ & $0.68 \%$ & $1.27 \%$ \\
\hline E & $0.70 \%$ & $0.90 \%$ & $1.54 \%$ \\
\hline
\end{tabular}


$M A E=\frac{1}{n} \sum_{i}^{n}\left|D A_{150 \text { RADIANCE }, i}-D A_{150 \text { RADIOSITY }, i}\right|$

Radiosity allows the calculation to be divided into 2 parts: a purely geometric part and a radiative exchange part. The geometric part consists in the calculation $\mathbf{F}$, which takes 6.5 minutes of computing time, taking advantage of the performance of GPU hardware (NVIDIA GTX 1070). The other part consists in solving Equation 7 to obtain $I$. The reflectivity properties of the patches only appear at this stage. With the proposed iterative resolution method for 9 reflections taken into account, the calculation time of this part is 13 minutes using CPU on a standard computer.

For this study, the aim is not to modify the geometry of this already built environment but to focus on changing the optical characteristics of materials. The purely geometric calculation of the view factors $\mathbf{F}$ only needs to be done once. In an only diffuse environment, just the second part of the calculation is repeated each time the diffuse reflectances are modified, allowing relatively short calculation times considering the large number of sensors. If specular reflectances are changed, the extended view factor $\mathbf{F}_{\text {eff }}$, and more specifically $\mathbf{S}$, have to be recalculated. The $\mathbf{S}$ calculation takes 25 minutes in this study (Table 2). The method is implemented in Matlab and all simulation times could be reduced after the implementation in a compiled language. In comparison, a simulation performed with RADIANCE using the 2-Phase method and the calculation parameters used for validation takes more than 12 hours using the 8 cores of the CPU of the same computer and the simulation have to be repeated each time reflectances are changed.

Table 2: Computation times for diffuse reflectance and specular reflectance modifications using radiosity.

\begin{tabular}{|c|c|c|c|}
\hline Modification & $\begin{array}{c}\text { Time for } \\
\text { S }\end{array}$ & $\begin{array}{c}\text { Time for } \\
I\end{array}$ & Total time \\
\hline Diffuse refl. & - & $13 \mathrm{~min}$ & $13 \mathrm{~min}$ \\
\hline Specular refl. & $25 \mathrm{~min}$ & $13 \mathrm{~min}$ & $38 \mathrm{~min}$ \\
\hline
\end{tabular}

Comparing the calculation times of different programs is a delicate and tricky task. These computation times can be rendered irrelevant by, for example, advances in computer hardware, or the use of cloud computing. This can change computation times showed in this paper for our method and RADIANCE. Each method has its own advantages and limitations and must be chosen according to the case studied. The method presented reaches its limits when a more complex reflection or transmission model is required, to take into account a higher level of detail of facade elements or openings. The method is restricted to surfaces that reflect or transmit in a perfectly diffuse and specular way, which nevertheless makes it possible to cover most of the existing scenarios in urban areas at a reasonable level of detail.

\section{Conclusion}

This work analyses the influence of facade reflectances in a dense urban environment on the daylight performance of buildings on a street scale. The interreflections between the surfaces represent a significant part of the total radiation received. Then, optical characteristics of the facade elements have an important impact on the performance of the surrounding buildings. The results show the potential of inter-reflections in the street to improve daylight conditions in the dimly-lit areas. The DA varies considerably for different usual facade compositions. The $\mathrm{DA}_{150}$ increases from $15 \%$ to $64 \%$ in the first floor spaces by changing the reflectance of the exterior walls from $20 \%$ to $80 \%$. This trend is the same for other DA threshold, $\mathrm{DA}_{300}$ change from $3 \%$ to $40 \%$. This simple fact justifies the interest of precisely considering the reflectance of the external environment during projects and can lead to the elaboration of urban planning rules to regulate the composition of facades in dense urban environments. Studying this urban problem from the inside of buildings, using metrics such as DA, provides accurate and rich information on building performance. Indeed, CBDM metrics rather than exterior-only studies give different and more relevant information in relation to the real needs of occupants. This highlights the interest and challenge of addressing daylight issues in urban areas in a multi-scale approach. The method based on the principle of radiosity allows studying a multitude of configurations with different facade compositions in short computation times with a large number of sensors (5348). Switching from one facade configuration to another requires 13 minutes of calculation in this study. The results obtained with the proposed method are in line with RADIANCE results with good calculation parameters for a building scale study. The Mean Absolute Error oscillates between $0.55 \%$ and $1.01 \%$ for the $\mathrm{DA}_{150}$ in the different cases studied.

\section{Acknowledgement}

This work was partially funded by the FSE_1_2017_1_144731 project from Agencia Nacional de Investigación e Innovación (ANII, Uruguay).

\section{References}

Acuña Paz y Miño, J., V. Lefort, C. Lawrence, \& B. Beckers. (2018). Maquette Numérique d'une rue du vieux Bayonne pour son étude thermique par éléments finis. In Teulier R. and Boutros N. A la pointe $d u$ BIM, Ingénierie \& architecture, enseignement \& recherche. Eyrolles. Paris (France).

Aguerre, J. P., E. Fernández, G. Besuievsky and B. Beckers. (2017). Computing Urban Radiation: A Sparse Matrix Approach. Graphical Models 91, 111.

Beckers, B. (2013). Taking Advantage of Low Radiative Coupling in 3D Urban Models. Proceedings from Eurographics Workshop on Urban Data Modelling and Visualisation. Girona (Spain), 2-5 May 2013. 
Brembilla, E., N. Drosou, \& J. Mardaljevic. (2016). Real World Complexity in Reflectance Value Measurement for Climate-Based Daylight Modelling. Proceedings from Building Simulation \& Optimisation (BSO 2016). Newcastle (UK), 12-14 September 2016.

CIBSE/SLL (2013). SLL Lighting Guide 9: Lighting for Communal Residential Buildings. Chartered Institution of Building Services Engineers. London (UK).

Cohen, M. F., \& D. Greenberg (1985). The Hemi-Cube: A Radiosity Solution for Complex Environments. Proceedings from SIGGRAPH '85. San Francisco (USA), 22-26 July 1985.

Compagnon, R. (2004). Solar and Daylight Availability in the Urban Fabric. Energy and Buildings 36(4), 321-328.

Crawley, D. B., J. W. Hand, \& L. K. Lawrie (1999) Improving the Weather Information Available to Simulation Programs. Proceedings from Building Simulation Conference 1999. Kyoto (Japan), 13-15 September 1999.

Dogan, T., C. F. Reinhart, \& P. Michalatos (2012). Urban Daylight Simulation Calculating the Daylit Area of Urban Designs. Proceedings from Fifth National Conference of IBPSA-USA. Madison (USA), 1-3 August 2012.

Fernández, E., B. Beckers and G. Besuievsky (2016). A Fast Daylighting Method to Optimize Opening Configurations in Building Design. Energy and Buildings 125: 205-218.

Geebelen, B., M. Van Der Voorden and H. Neuckermans (2005). Fast and Accurate Simulation of Long-Term Daylight Availability Using the Radiosity Method. Lighting Research \& Technology 37(4), 295-310.

Goral, C. M., K. E. Torrance, D. P. Greenberg, \& B. Battaile (1984). Modeling the Interaction of Light between Diffuse Surfaces. Proceedings from SIGGRAPH '84. Minneapolis (USA), 23-27 July 1984.
IESNA (2012). IES LM-83-12, Approved Method: IES Spatial Daylight Autonomy (SDA) and Annual Sunlight Exposure (ASE).

Mardaljevic, J. (2012). Multiscale Daylight Modeling for Urban Environments. In Beckers, B. Solar energy at urban scale. John Wiley \& Sons. Hoboken (USA).

Paule, B., Boutillier J., \& Pantet S. (2015). Shading Device Control: Effective Impact on Daylight Contribution. Proceedings from CISBAT, Future Buildings and Districts - Sustainability Form Nano to Urban Scale. Lausanne (Switzerland), 9-11 September 2015.

Perez, R., R. Seals and J. Michalsky. (1993). AllWeather Model for Sky Luminance DistributionPreliminary Configuration and Validation. Solar Energy 50(3), 235-245.

Rushmeier, H E. and K. E. Torrance (1990). Extending the Radiosity Method to Include Specularly Reflecting and Translucent Materials. $A C M$ Transactions on Graphics 9(1), 1-27.

Sillion, F. X., \& C. Puech (1989). A General Two-Pass Method Integrating Specular and Diffuse Reflection. Proceedings from SIGGRAPH '89. Boston (USA), 31 July - 4 August 1989.

Strømann-Andersen, J. and P. A. Sattrup (2011). The Urban Canyon and Building Energy Use: Urban Density versus Daylight and Passive Solar Gains. Energy and Buildings 43(8), 2011-2020.

Tregenza, P. R. and I. M. Waters (1983). Daylight Coefficients. Lighting Research \& Technology 15(2), 65-71.

Tregenza, P. R. (1987). Subdivision of the Sky Hemisphere for Luminance Measurements. Lighting Research \& Technology 19(1), 13-14.

Ward, G. J. (1994). The RADIANCE Lighting Simulation and Rendering System. Proceedings from SIGGRAPH '94. Orlando (USA), 24-29 July 1994. 